\title{
Disrupted apical exocytosis of cargo vesicles causes enteropathy in FHL5 patients with Munc18-2 mutations
}

\author{
Georg F. Vogel, ${ }^{1,2}$ Jorik M. van Rijn, ${ }^{3}$ Iris M. Krainer, ${ }^{1,2}$ Andreas R. Janecke, ${ }^{1}$ Carsten Posovszky, ${ }^{4}$ \\ Marta Cohen, ${ }^{5}$ Claire Searle, ${ }^{6}$ Prevost Jantchou, ${ }^{7}$ Johanna C. Escher, ${ }^{8}$ Natalie Patey, ${ }^{6}$ Ernest Cutz, ${ }^{9}$ \\ Thomas Müller, ${ }^{1}$ Sabine Middendorp, ${ }^{3}$ Michael W. Hess, ${ }^{10}$ and Lukas A. Huber ${ }^{2}$ \\ 'Department of Paediatrics I and 'Division of Cell Biology, Medical University of Innsbruck, Innsbruck, Austria. ${ }^{3}$ Division of \\ Paediatrics, Department of Paediatric Gastroenterology and Regenerative Medicine Center Utrecht, Wilhelmina Children's \\ Hospital, University Medical Centre (UMC) Utrecht, Utrecht, The Netherlands. ${ }^{4}$ Department of Pediatrics and Adolescent \\ Medicine, University Medical Center Ulm, Ulm, Germany. ${ }^{5}$ Sheffield Children's Hospital NHS Trust, Western Bank, \\ Sheffield, United Kingdom. ${ }^{6} \mathrm{Clinical}$ Genetics, Nottingham University Hospitals NHS Trust, Nottingham, United Kingdom. \\ ${ }^{7}$ Castroentérologie Hépatologie et Nutrition Pédiatrique Hôpital Sainte-Justine, Université de Montréal, Montréal, Quebec, \\ Canada. ${ }^{8}$ Department of Pediatric Gastroenterology, Sophia Children's Hospital, Erasmus MC, Rotterdam, \\ The Netherlands. ${ }^{9}$ The Hospital for Sick Children, Toronto, Canada. ${ }^{10}$ Division of Histology and Embryology, Medical \\ University of Innsbruck, Innsbruck, Austria.
}

Familial hemophagocytic lymphohistiocytosis 5 (FHL5) is an autosomal recessive disease caused by mutations in STXBP2, coding for Munc18-2, which is required for SNARE-mediated membrane fusion. FHL5 causes hematologic and gastrointestinal symptoms characterized by chronic enteropathy that is reminiscent of microvillus inclusion disease (MVID). However, the molecular pathophysiology of FHL5-associated diarrhea is poorly understood. Five FHL5 patients, including four previously unreported patients, were studied. Morphology of duodenal sections was analyzed by electron and fluorescence microscopy. Small intestinal enterocytes and organoid-derived monolayers displayed the subcellular characteristics of MVID. For the analyses of Munc18-2-dependent SNARE-protein interactions, a Munc18-2 CaCo2-KO model cell line was generated by applying CRISPR/Cas9 technology. Munc18-2 is required for SIp4a/ Stx3 interaction in fusion of cargo vesicles with the apical plasma membrane. Cargo trafficking was investigated in patient biopsies, patient-derived organoids, and the genome-edited model cell line. Loss of Munc18-2 selectively disrupts trafficking of certain apical brush-border proteins (NHE3 and CLUT5), while transport of DPPIV remained unaffected. Here, we describe the molecular mechanism how the loss of function of Munc18-2 leads to cargo-selective mislocalization of brush-border components and a subapical accumulation of cargo vesicles, as it is known from the loss of polarity phenotype in MVID.

Conflict of interest: The authors have declared that no conflict of interest exists.

Submitted: April 14, 2017

Accepted: June 6, 2017

Published: July 20, 2017

Reference information: JCI Insight. 2017;2(14):e94564. https://doi.org/10.1172/jici. insight.94564.

\section{Introduction}

Familial hemophagocytic lymphohistiocytosis (FHL) is a rare, autosomal recessively inherited disorder, with a heterogeneous genetic background. Patients with FHL typically present with episodes of prolonged fever, hepatosplenomegaly, lymphadenopathy accompanied by pancytopenia, and hyperferritinemia (1-4). Patients' activated natural killer cells and cytotoxic $\mathrm{T}$ lymphocytes show reduced or absent ability to kill virus-infected cells. As this results in hyperactivation and ineffective immune response at the same time, it can lead to patient death if not overcome by an immunosuppressive regime $(5,6)$. However, a definitive cure of this genetic disease can only be achieved by hematopoietic stem cell transplantation (HSCT) (7).

So far, 5 types of FHL have been classified based on mutations in different genes: HPHL1 (FHL1) (8), PRF1 (FHL2) (9), UNC13D (FHL3) (10), STX11 (FHL4) (2), and STXBP2 (FHL5) (3, 4). With the exception of HPHL1, the function of the genes involved has been identified and been linked to transport, membrane fusion, and exocytosis of perforin-containing lytic granules. This seems to be the molecular defect underlying FHL and results in the inability of lymphocytes and macrophages to efficiently kill pathogens and infected cells. 
Although FHL is primarily characterized as a defect of the immune system, FHL5 patients display additional intestinal symptoms, such as severe noninflammatory diarrhea, which persists after HSCT $(11,12)$. A recent study reported five patients with FHL5 and gastrointestinal symptoms (chronic diarrhea, gastroesophageal reflux, and abdominal pain) displaying ultrastructural features reminiscent of microvillus inclusion disease (MVID) (12). MVID is a rare, congenital enteropathy, which is also inherited autosomal recessively $(13,14)$. Typically, days to weeks after birth, neonates present with severe noninflammatory diarrhea and metabolic acidosis (14-16). Many patients die within the first few years of life. They depend on total parenteral nutrition, and, at present, small bowel transplantation is the only curative therapy. Ultrastructure analysis - the gold standard of MVID diagnosis shows partial or complete loss of the enterocytes' brush-border microvilli, intracellular vacuoles with microvilli on the inside (microvillus inclusions [MI], ref. 14), and/or ectopic basolateral microvilli (17, 18). Moreover, enterocytes regularly display subapical accumulations of periodic acid-Schiff-positive (PAS-positive) vesicles and tubules (historically termed secretory granules, ref. 13, 14), recently identified as aberrant Rab11-Rab8-positive recycling compartments (17). Genetic analysis revealed that mutations in the MYOB5 or STX3 genes are causative for MVID (18-21).

Correct targeting of cargo vesicles to the destined plasma membrane is pivotal for specific cellular processes. In polarized epithelial cells, for example, apical cargo (e.g., enzymes, receptors, transmembrane transporters) is delivered via a cascade of Rab11a and Rab8a (22). At the plasma membrane, the octameric exocyst complex tethers cargo vesicles to the plasma membrane and thus facilitates fusion via the interaction of cognate vesicular and target soluble NSF attachment protein receptors (v-SNARE and t-SNARE, respectively). The v-SNARE-like protein was identified to be synaptotagmin-like protein 4a (Slp4a), whereas syntaxin 3 (Stx3) serves as the corresponding t-SNARE (23). In natural killer cells and cytotoxic T lymphocytes, exocytosis of cytotoxic granules follows a very similar mechanism. Here, the fusion of exocytic vesicles is performed by the v-SNARE Vamp7 and the t-SNARE syntaxin 11 (Stx11) (24). The v-/t-SNARE interaction is mediated by the Sec1/Munc18-like protein (SM) Munc18-2/Stxbp2 (25) (referred to as Munc18).

We have recently added further mechanistic understanding of the pathophysiology of MVID. Apical transmembrane transporters (e.g., NHE3, GLUT5, CFTR), relevant for proper physiological function of enterocytes, are dependent on of myosin $\mathrm{Vb}$ (Myo5B), Rab11a, and Rab8a for transport. At the apical membrane, the cargo vesicles are dependent on Slp4a, Munc18, Vamp7, and Stx3 in order to dock and fuse (26). In case of MVID, when either Myo5B or Stx3 are lost, actin motor transport or membrane fusion of cargo vesicles is disrupted, which might result in a cargo-selective mislocalization of relevant transporters (26). The precise molecular mechanism underlying FHL5 enteropathy, and the similarity to MVID, was not understood.

In the present study, we report five patients, including four patients not previously reported with mutations in $S T X B P 2$, suffering from a congenital enteropathy, highly reminiscent of MVID. We have investigated FHL5 patient material and a de novo-generated Munc18-2-KO CaCo2 model enterocyte cell line by the use of CRISPR/Cas9 genome editing (27). Here, we demonstrate that Munc18-2 is required for Slp4a/ Stx3-mediated fusion of apical cargo vesicles. Therefore, loss of function of STXBP2 leads to cargo-selective mislocalization of certain apical cargo (e.g., NHE3 and GLUT5) and a subapical accumulation of cargo vesicles, as it is known from MVID caused by MYO5B or STX3 mutations.

\section{Results}

Patient description. For this study, we recruited five patients.

Patient 1, a female, was born in 2011 in Sheffield, United Kingdom, to consanguineous Pakistanian parents (first cousins). There were two healthy brothers. An echogenic bowel was seen on the prenatal ultrasound, and no other problems were noted. The patient suffered from congenital diarrhea since the first days of life and failed to thrive. Severe diarrhea ameliorated when getting nil by mouth but recurred 2 days after any feed. A homozygous STXBP2 mutation, c.37+1G>A, affecting the canonical donor splice site of intron 1 and being absent from EXAC and dbSNP databases was identified in the patient, establishing the diagnosis of FHL5. Within the first year of life she underwent allogenic stem cell transplantation for hemophagocytic lymphohistiocytosis (HLH). Afterward, she required total parental nutrition. The patient died at the age of 13 months of unknown cause that was supposedly related to disease after transplant. Sequencing the whole-coding region and flanking splice sites revealed no concomitant mutations in the 
MYO5B or STX3 genes. At the MYO5B locus, heterozygosity for 8 common SNPs and a common 3-bp indel variant were observed, demonstrating that the patient was heterozygous at this locus. Given the parental consanguinity and the negative sequence analysis, the results from direct and indirect DNA testing likely exclude MYO5B from playing a role in the disease pathogenesis in this patient. There were no private or rare variants seen in the other MVID gene STX3.

Patient 2, a male, was reported previously (12). He was born to healthy consanguineous Turkish parents. Two older siblings had died from HLH disease in their first years of life. His twin brother and an older sister were apparently healthy. He developed diarrhea, pancytopenia, and fever, with signs of sepsis and disseminated intravascular coagulation without the identification of an infectious agent at the age of 3 weeks. Bone marrow morphology revealed hemophagocytosis. He received a HSCT from his HLA-matched older healthy sister at the age of 6 months. Targeted sequence analysis of the STXBP2 gene revealed a homozygous 3-bp deletion in exon 9 (c.693_695delGAT; p.Ile232del) completely hampering the binding of Munc18 to Stx11, as described previously (3). Later, exome sequencing confirmed the presence of this $S T X B P 2$ mutation and indicated the presence of a $50 \%$ degree of chimerism. There were no private or rare variants seen in the MVID genes MYO5B and STX3.

Patient 3, a male, was born at term in 2014 as the second child of nonconsanguineous parents. His older brother was healthy. In the first week of life, he developed feeding problems, vomiting, and profuse watery diarrhea. During the first weeks, watery diarrhea persisted, and he was hospitalized three times because of dehydration, weight loss, and metabolic acidosis, despite elemental formula. Total parenteral nutrition was started, and an upper gastrointestinal endoscopy was performed at week 6 because of clinical suspicion of MVID. Duodenal biopsies revealed an intact brush-border membrane of the duodenum but subtle chronic inflammation, with microgranulomas in stomach and duodenum. At week 7 , he deteriorated (fever, hepatosplenomegaly, pancytopenia, increased liver enzymes and ferritin) and was suspected of HLH. Treatment was started with dexamethasone, cyclosporin, and etoposide, with improvement of laboratory results as well as better tolerance of enteral feeding. Genetic analysis showed a homozygous c. $902+5 \mathrm{G}>$ A mutation in STXBP2, consistent with FHL type 5 (28). At 4 months of age he had an uncomplicated allogeneic HSTC (his brother was donor). Six months after the HSCT, he again developed feeding problems with vomiting, diarrhea, and failure to thrive. Enteral feeding by nasogastric tube was not tolerated, and a nasoduodenal tube had to be placed.

Duodenal biopsies were repeated at age 1.5 years, showing no specific changes in histology or electron microscopy (EM) of the duodenal mucosa. Currently, he is 2.5 years old, is able to tolerate a minimal amount of oral food, and has diarrhea 3 times per day. He has longitudinal growth failure, with current height for age at $-2.5 \mathrm{SD}$ and weight for height $+1.5 \mathrm{SD}$. He is receiving $1,000 \mathrm{ml}$ amino acid formula via duodenal tube as well as sodium bicarbonate, sodium chloride, phosphate, esomeprazole, and cholestyramine.

Patient 4, a male, was born in 2006 as the second child of consanguineous parents (first cousins) at 35 weeks of gestation following elected induction for polyhydramnios. The older sister is healthy. Following birth, he presented with chronic diarrhea, weight loss and failure to thrive, dehydration and gastroesophageal reflux. FHL developed at 3.5 month of age. Genetic analysis revealed a homozygous c.1146delC mutation in $S T X B P 2$, which predicts a truncation of the gene product, p.(Lys383Argfs*4). Total parenteral nutrition was initiated at 6 months of age. HSCT was performed in February 2007 in Montreal. Major subsequent complications included cytomegalovirus infection and late graft-versus-host disease. The severe diarrhea persisted after transplantation. The patient has depended on parenteral nutrition since infancy (6 month of age) and tolerates small amount of feeds. Stool output is high at 1.8-2 1/d (measured in 2015 during hospital admission). Output has improved following diet modifications to $700-1,200 \mathrm{ml} / \mathrm{d}$, but, currently, the oral intake is not significant, partly due to feeding disorders in relation with long-term total parenteral nutrition. Prolonged parenteral nutrition complications include central vein thrombosis and recurrent episodes of line sepsis. Intestinal transplantation has been repeatedly considered in this patient but was not performed until his current age of 10 years.

Patient 5 is the brother of patient 4. He was born in May 2016 at 36 weeks of gestation. Antenatal ultrasounds displayed enlarged intestines that were full of fluids. As early as 7 days after birth, he developed severe watery diarrhea, resulting in hospitalization. Because of the brother's history, we immediately suspected that the diarrhea was linked to an intestinal manifestation of FLH. Thus, genetic analysis was performed and identified the same mutation as in his elder brother within STXBP2. During the subsequent weeks, he developed two severe episodes of macrophage activation syndrome that were treated with intravenous steroids and 
A

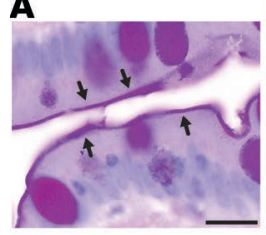

D

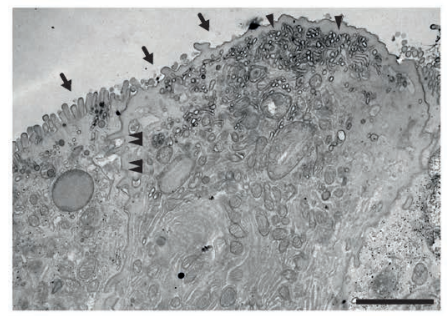

$\mathbf{F}$

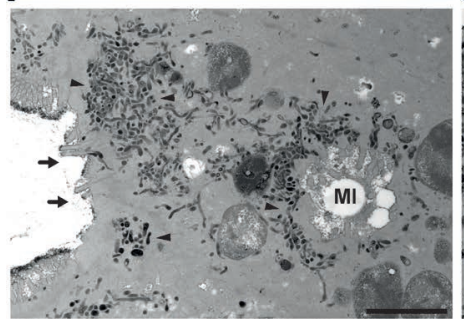

B

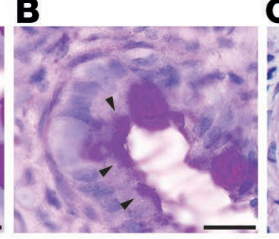

E

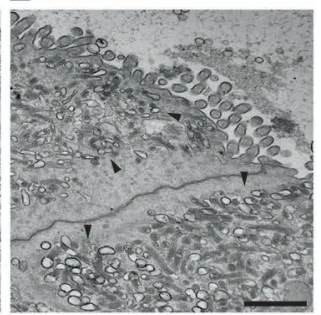

G

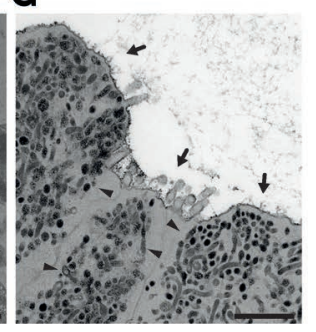

Figure 1. Duodenal enterocyte ultrastructure in FHL5 appears identical to MVID. (A-C) Light micrographs of duodenal sections from a healthy control patient (A), patient 1 (B), and patient 2 (C); PAS-positive brush border (arrows in $\mathbf{A}$ ) and subapical secretory granules are indicated (arrowheads in $\mathbf{B}$ and $\mathbf{C}$ ). (D and E) Electron micrographs of villus enterocytes from patient 1 show shortening or loss of apical microvilli (arrows), subapical accumulation of partly PAS-positive vesicles and tubules (arrowheads), and ectopic lateral microvilli (double arrowheads). (F and $\mathbf{G ) ~ V i l l u s ~ e n t e r o c y t e s ~ o f ~ p a t i e n t ~} 2$ show shortening or loss of apical microvilli (arrows), subapical accumulation of PAS-positive vesicles and tubules (arrowheads), and intracellular microvillus inclusions (MI). Scale bar: $20 \mu \mathrm{m}$ (A-C); $2 \mu \mathrm{m}$ (D and $\mathbf{F}) ; 1 \mu \mathrm{m}$ (E and $\mathbf{G}$ ).

cyclosporin. The patient is currently dependent on total parenteral nutrition, with only small amounts of milk. On this regiment, the diarrhea remains substantial (3-6 bowel movements a day), but weight gain is appropriate. HSCT was discussed with the parents and performed in January 2017.

In summary, we report five FHL5 patients, of which four represent previously unreported cases, with mutations in STXBP2. All five patients manifest with severe, watery, MVID-like diarrhea, in addition to hematologic symptoms.

FHL5 patient enterocytes display MVID morphology. As the patients reported herein suffered from congenital enteropathy, and a previous report about patient 2 and three other patients (12) indicated subcellular alterations of duodenal enterocytes reminiscent of MVID, we next carried out a detailed morphological analysis.

Comparative light microscopy of duodenal biopsies from a healthy control (Figure 1A) and patients 1 and 2 (Figure 1, B and C) showed aberrant intracellular PAS staining in the patient samples. EM revealed a subcellular phenotype of the enterocytes virtually indistinguishable from MVID. In patients 1, 2, and 4,shortening or complete loss of apical microvilli of villus enterocytes was observed (Figure 1, D-G, and Supplemental Figure 1A; supplemental material available online with this article; https://doi.org/10.1172/jci.insight.94564DS1). MI were found in patients 2 and 4 (Figure $1 \mathrm{~F}$ and Supplemental Figure 1A) and basolateral microvilli were found in patients 1 and 4 (Figure 1D). Furthermore, clusters of subapical vesicles and tubules characterized the subapical cytoplasm of enterocytes from patients 1, 2, and 4 (Figure 1, D-G, and Supplemental Figure 1A). These structures were heterogeneous in size, staining intensity, and PAS reactivity (Figure 1, D-G) and frequently surrounded cytoplasmic MI (Figure 1F).

Organoid-derived, fully polarized enterocyte monolayer cultures were generated from a control patient and FHL5 patient 3 (Figure 2). In contrast to the control sample (Figure 2B), the subapical cytoplasm of enterocytes from patient 3 contained numerous spherical to rod-shaped PAS-positive vesicles (Figure 2C) resembling MVID-specific "secretory granules."

In MVID patients with mutated $M Y O 5 B$ or $S T X 3$, Myo5B KO mice, and MVID model cell lines, a translocation of the apical recycling endosomal marker Rab11a was observed (26, 29-31). Thus, we further studied the localization of Rab11a in natively frozen duodenal sections by confocal immunofluorescence laser scanning microscopy. Indeed, Rab11 showed a disturbed and increased cytoplasmic localization in FHL5 when compared with the predominantly subapical staining in healthy controls (Figure 3A). Additionally, the localization of the apical t-SNARE Stx3 was similarly disturbed in enterocytes of FHL5 patient 2 (Figure 3B). Interestingly, mislocalized Stx3 and Rab11 staining was also seen around and/or within MI (Figure 3). This has also been reported before in the murine Myo5B KO MVID model (31) and further emphasizes the notion of MI being intracellular de novo apical domains.

In conclusion, the ultrastructural characteristics of duodenal enterocytes from the FHL5 patients presented herein appeared identical to those from reported MVID patients (17, 32). All major morphological hallmarks of MVID were found: first, loss or shortening of apical microvilli; second, a subapical accumulation of PAS-positive vesicles and tubules; and, finally, MI and/or basolateral microvilli.

Targeted disruption of Munc18 recapitulates intestinal features of FHL5. In order to analyze the molecular mechanism underlying MVID-like enteropathy associated with FHL5, we first generated a disease model cell line. For this, we used $\mathrm{CaCo} 2$ cells, a human enterocyte model cell line, which is able to establish enterocyte polarity in culture (26). Using CRISPR/Cas9-mediated genome editing, we 
A

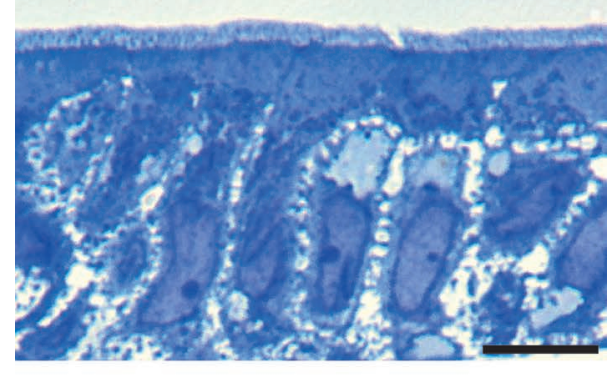

\section{B}

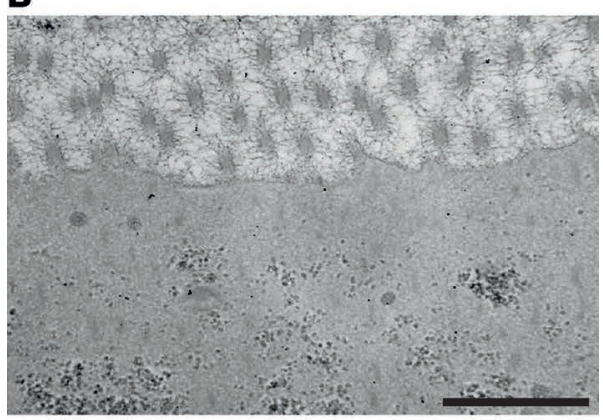

C

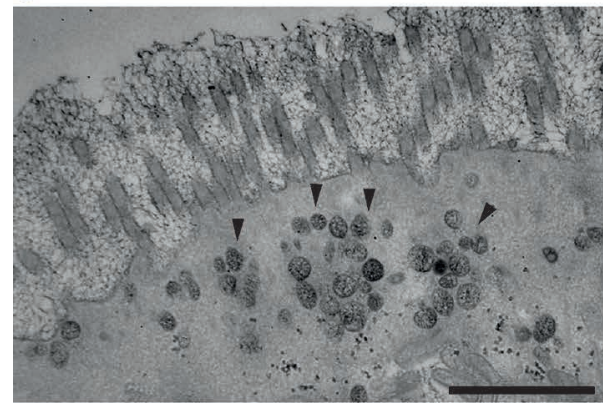

Figure 2. Fine structure of differentiated organoid-derived monolayers. (A) Monolayer culture derived from organoids from a duodenal biopsy of a control patient. Enterocytes show the typical morphology of fully polarized epithelial cells (light micrograph of a Toluidine blue-stained semithin section). (B) Electron micrograph of an organoid-derived monolayer from a control patient showing normal PAS staining, confined to intracellular glycogen and the glycocalix. Apical microvilli are cut transversally. (C) Electron micrograph of an organoid-derived enterocyte monolayer from FHL patient 3 shows a subapical accumulation of PAS-positive vesicles and virtually normal microvilli. Scale bar: $10 \mu \mathrm{m}(\mathbf{A}) ; 1 \mu \mathrm{m}$ (B and $\mathbf{C})$.

specifically targeted the $S T X B P 2$ locus. This resulted in a complete loss $-\mathrm{KO}-$ of Munc18 protein level (Figure 4A). To control for potential off-target effects by genome editing, we reintroduced a HA-Strep-TactinII-tagged (HS-tagged) version of WT Munc18 (HS-Munc18_gR) and the reported mutation/deletion of FHL5 patient 2 (HS-Munc18_gR-d232) in Munc18-KO cells (Figure 4A). The 232del mutant was exemplarily chosen for further mechanistic studies. Both $\mathrm{cDNAs}$ harbor additional noncoding mutations, rendering them resistant to the targeting sequence used for CRISPR/Cas9. Since loss of enterocyte polarity is a feature of intestinal FHL5, we subjected the model cell lines to cyst formation, an assay for challenging the establishment of epithelial polarity (26). Indeed, loss of Munc18 resulted in a significantly decreased single central lumen formation in comparison with the WT CaCo2 cells. This effect could be reverted upon the reintroduction of the HS-Munc18_gR. However, HS-Munc18_gR-d232 failed to revert the phenotype (Figure 4, B and C).

To analyze the morphology in greater detail, WT, KO, and reverted cells were grown on porous membrane supports in order to establish enterocyte polarity. In KO cells, EM revealed a reduction and shortening of apical microvilli and an aberrant accumulation of subapical vesicles (Figure 4D). The detected ultrastructural aberrations were specific for the loss of Munc18, as the reverted cell line displayed a regular enterocyte ultrastructure (Figure 4E).

Altogether, loss of Munc18 in $\mathrm{CaCo} 2$ cells resulted in disruption of enterocyte polarity and yielded a subcellular phenotype highly comparable to FHL5-linked MVID.

Munc18 is pivotal for SNARE pairing at the apical plasma membrane. Munc18 was shown to mediate the Vamp7/Stx11 (25) and Slp4a/Stx3 interaction (33). In MVID, the Stx3/Munc18 interaction is strongly reduced as a consequence of loss of Myo5B (26), ultimately leading to disrupted exocytosis of cargo vesicles. Therefore, we hypothesized that loss of Munc18 function in FHL5 would result in disturbed cognate t-/v-SNARE interaction in enterocytes. To test this, we expressed HS-tagged Slp4a, a v-SNARE-like protein, and Stx3 in Munc18 WT and KO (gMunc18) $\mathrm{CaCo} 2$ cells and performed coaffinity purification experiments. This method is based on the strong affinity of the Strep-TactinII tag to Strep-Tactin on Sepharose beads, which allows purification of the bait proteins and their interaction partners from cellular lysates (34). Indeed, HS-Slp4a copurified with Stx3, as previously reported (26), as well as Munc18, in WT cells (Figure 5A). Upon loss of Munc18, however, interaction of Slp4a and Stx3 was abolished (Figure 5A). Reciprocally, Stx3/Slp4a interaction was disrupted when HS-Stx3 was purified (Figure 5B). Furthermore, Rab11a, a bona fide marker for subapical exocytic/recycling compartments and an interaction partner of Stx3 in WT cells (26) did not copurify with Slp4a when Munc18 was deleted (Figure 5B). Overall, Stx3 protein levels remained unaffected upon the deletion of Munc18 (Figure 5, A and B); however, the subcellular distribution was altered similarly to that seen in FHL5 patients, with increased intracellular Stx3 localization (Supplemental Figure 1B). This might contribute to the observed trafficking defect.

In order to test the effect of the STXPB2 Ile232del mutation on the physiological function of Munc18-mediated SNARE binding, we purified HS-Munc18_gR and HS-Munc18_gR-d232 from KO cells and studied known SNARE interactions (Figure 5C). The deletion of isoleucine 232 of the Munc18 protein sequence has been reported to hamper binding to Stx11 (3). Both Slp4 and Stx3 copurified with WT Munc18. By contrast, no copurification of the Ile232del mutant with Slp4a or Stx3 was detectable (Figure 5C).

These results indicate a pivotal role of Munc18 for cognate t-/v-SNARE interaction and subsequent membrane fusion, e.g., cargo vesicles/Slp4a and the apical plasma membrane/Stx3. In respect to this, the Ile232del mutant of Munc18 rendered the protein dysfunctional regarding v-/t-SNARE-mediated membrane fusion.

Loss of Munc18 selectively disrupts apical cargo trafficking. In MVID, disturbed interaction of Slp4a/ Stx3 in apical fusion processes was previously shown to impair the trafficking and localization of apical 
A
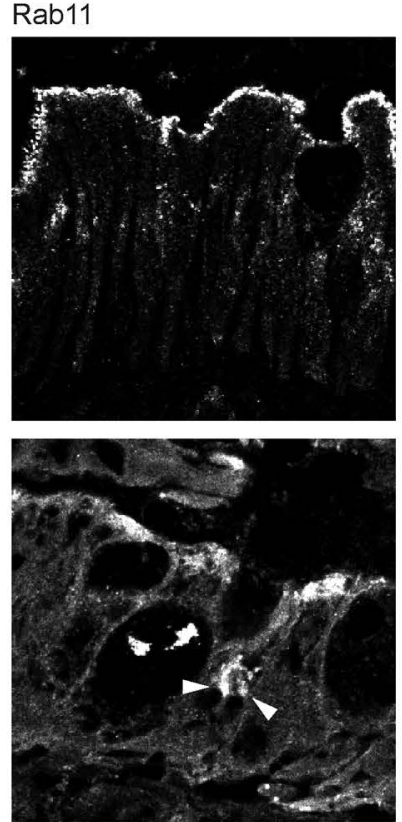

B
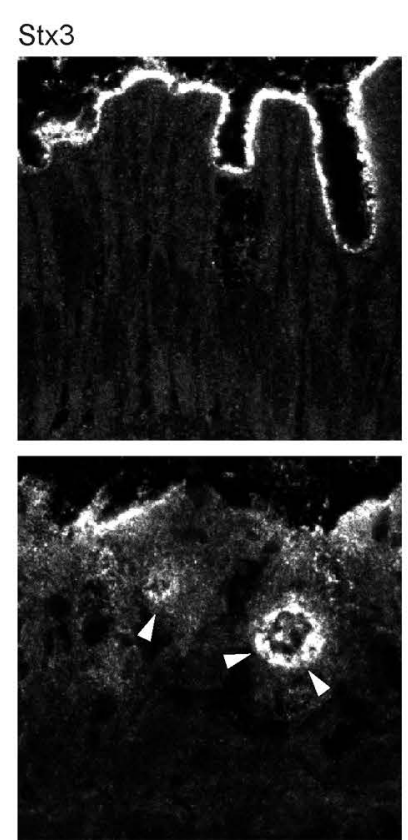

Actin
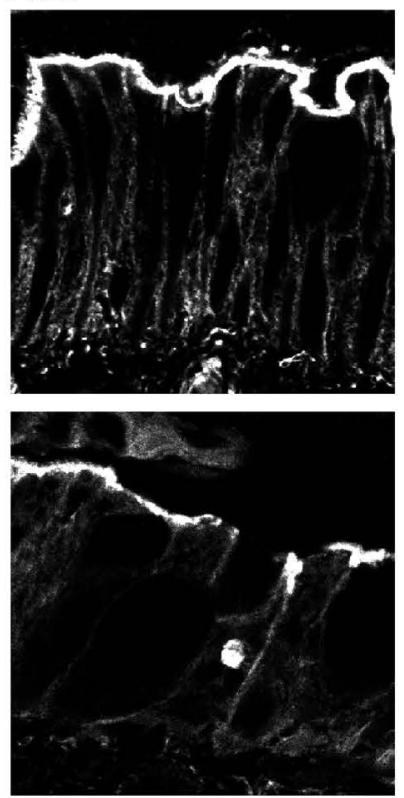

Actin
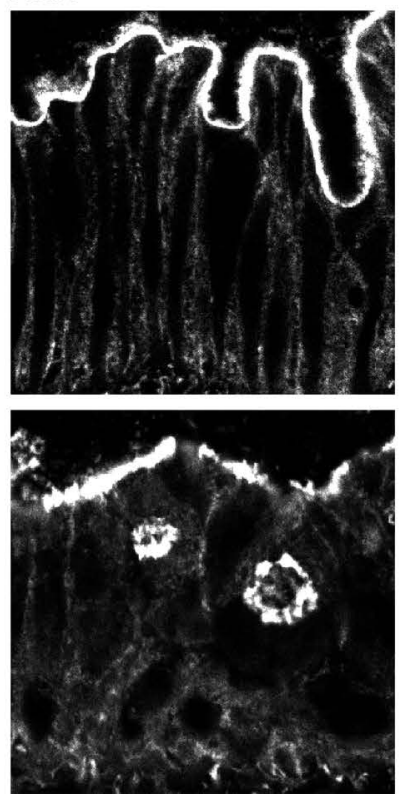

Rab11/Actin/Nuclei
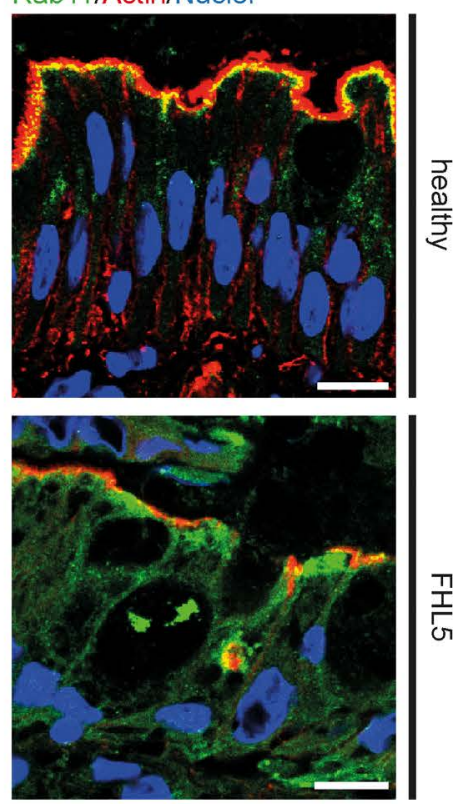

Stx3/Actin/Nuclei
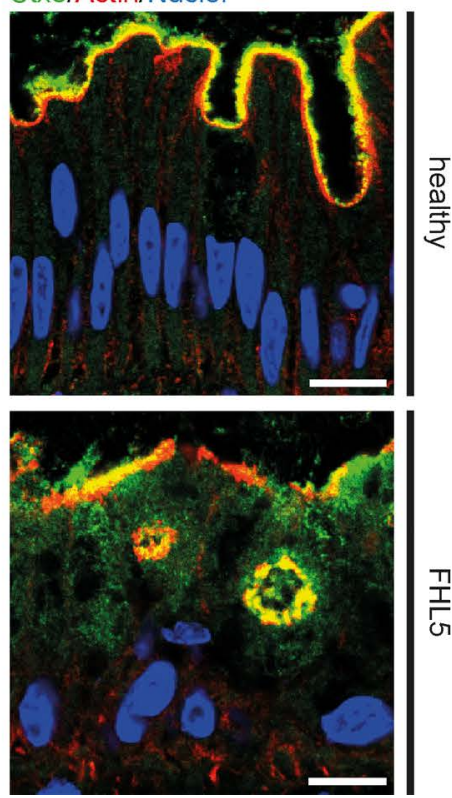

Figure 3. Aberrant distribution of apical proteins in FHL5. Laser-scanning immunofluorescence micrographs of duodenal enterocytes of patient 2 show (A) mislocalization of subapical Rab11 to the cytoplasm and accumulation around microvillus inclusions (white arrowheads) in contrast to controls and (B) mislocalization of apical Stx3 to the cytoplasm in association with microvillus inclusions (white arrowheads) in comparison to controls. (A and B) Brush-border microvilli are indicated by actin staining. Scale bar: $10 \mu \mathrm{m}$.

transmembrane transporters in enterocytes (26), e.g., sodium-hydrogen exchanger 3 (NHE3) and glucose transporter 5 (GLUT5), which are relevant for enterocyte physiology (35-37). Therefore, we further analyzed the localization of these proteins in Munc18-KO cells. Porous filter supports were used in order to allow establishment of enterocyte monolayer polarity. Indeed, loss of Munc18 resulted in a redistribution of normally apically destined NHE3 and GLUT5 proteins to a diffuse cytoplasmic localization (Figure 6, A and B). However, the localization of the brush-border enzyme dipeptidylpeptidase 4 (DPPIV) remained unaffected at the apical plasma membrane (Figure 6B).

Analogously, we analyzed the distribution of NHE3 and DPPIV in FHL5 duodenal biopsies. In 
A

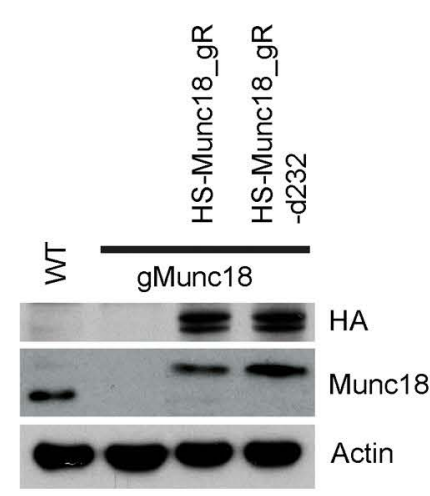

B

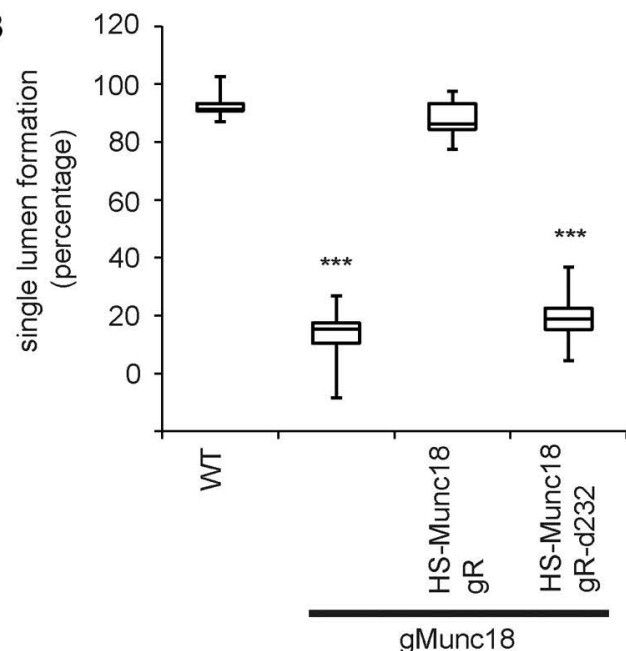

C
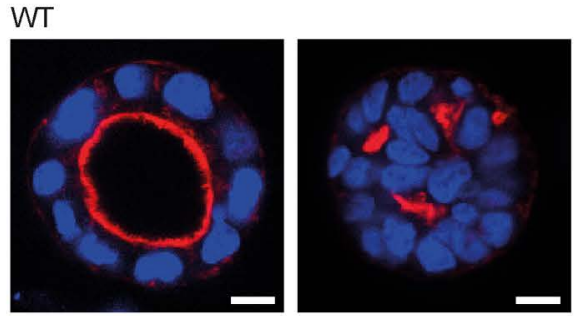

HS-Munc18_gR

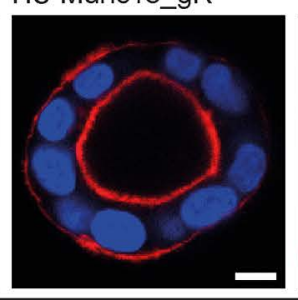

HS-Munc18_gR-d232

gMunc18

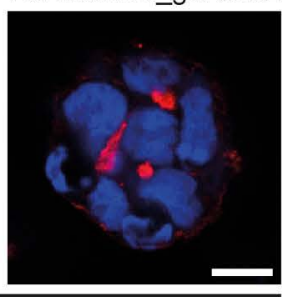

E

D

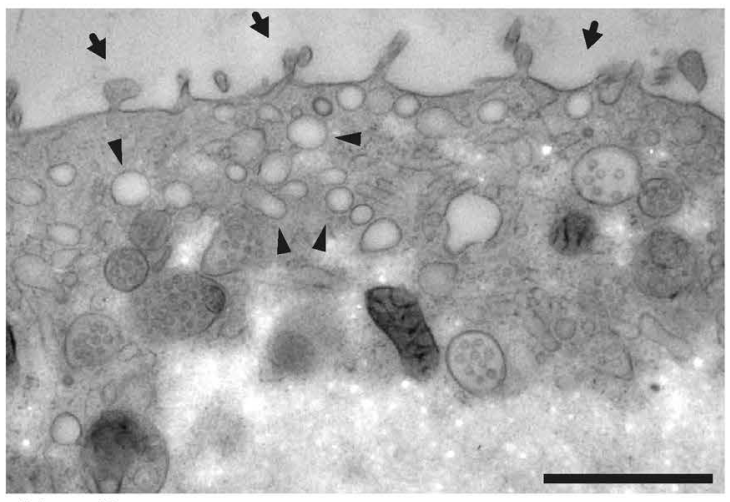

gMunc18
E

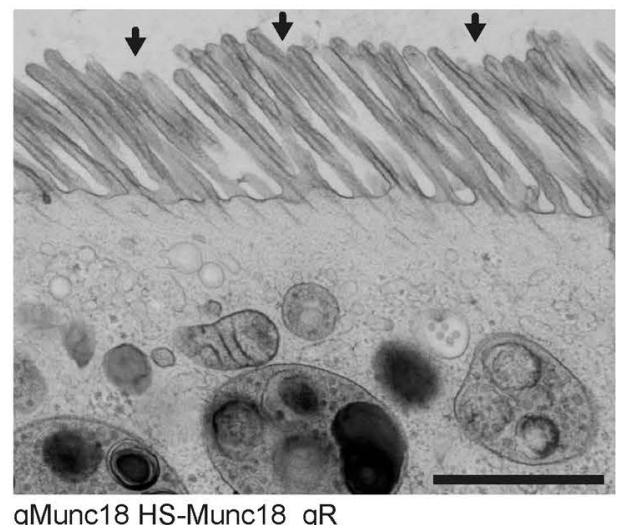

Figure 4. Loss of Munc18 disrupts enterocyte polarity. (A) Western blot showing CaCo2 WT, Munc18-KO (gMunc18), and Munc18-KO cells reverted with HS-tagged, gRNA-resistant Munc18 WT and d232 mutant. (B) Quantification of single lumen formation (bounds of boxes represent 25th/75th percentiles, lines within boxes indicate medians, and whiskers represent minimum/maximum; statistical significance was tested by Student's $t$ test; ${ }^{* * *} P<0.005, n \geq$ 100 cysts/experiment, 3 independent experiments). (C) Polarity is disrupted upon Munc18 depletion (gMunc18) in a 3D cyst formation assay in comparison to WT. Reexpression of HS-Munc18_gR reverts the phenotype, while HS-Munc18_gR-d232 does not. Actin is labeled in red an nuclei in blue; basal actin stain is seen in reaction to extracellular matrix (e.g., Matrigel). (D) Loss of Munc18 results in loss of apical microvilli (arrows) and subapical vesicle accumulation (arrowheads) in CaCo2 cells. Scale bar: $1 \mu \mathrm{m}$. (E) Reexpression of HS-Munc18_gR reverts the Munc18-KO phenotype. The brush-border microvilli are indicated with arrows. Scale bar: $10 \mu \mathrm{m}$ (C); $1 \mu \mathrm{m}$ (D).

patient 2, immunofluorescence microscopy showed NHE3 throughout the cytoplasm in contrast to agematched controls, whereas DPPIV was found at the brush border in the FHL5 patient and control samples (Figure 6C). Complementary immuno-EM investigation of samples from FHL5 patients 1, 2, and 4 identified NHE3 at the subapically clustering vesicles and tubules (Supplemental Figure 2, A-C) and, to a minor extent, at the apical microvilli and the endoplasmatic reticulum. Moreover, Rab11 and Stx3 


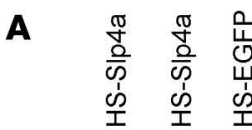

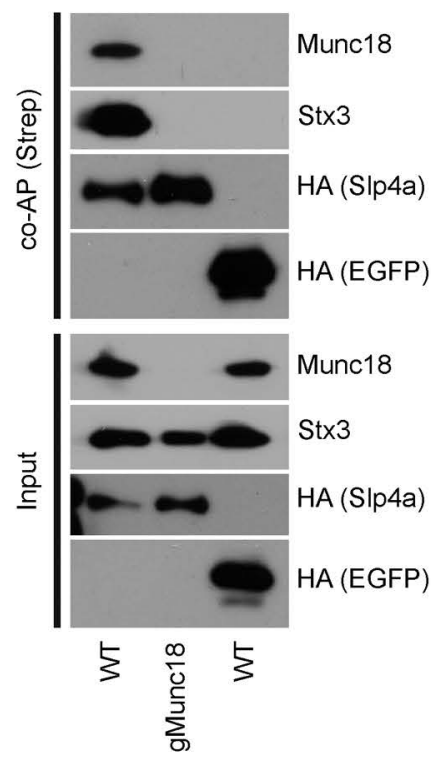

C

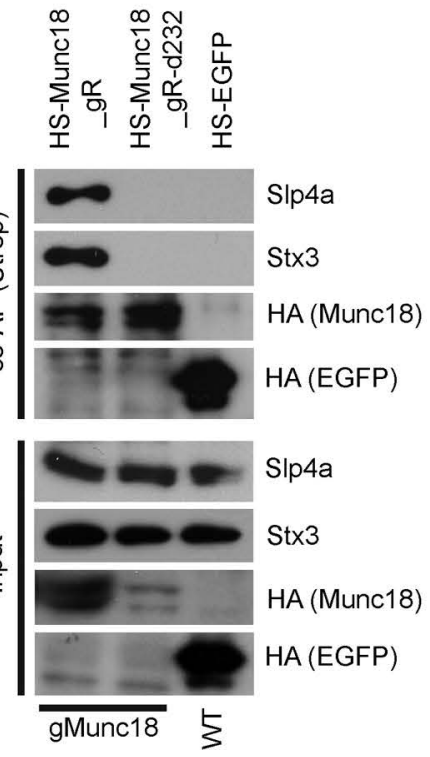

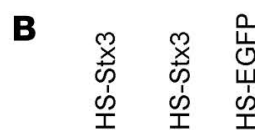
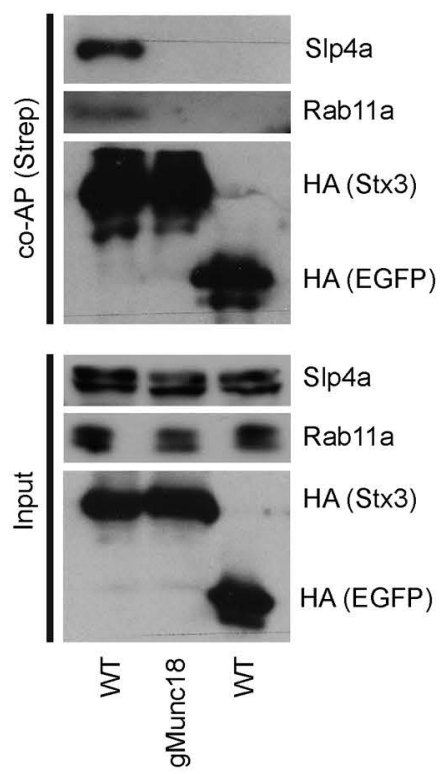

Figure 5. Munc18 is required for v-/t-SNARE interaction. (A) The interaction between the $v$-SNARE SIp4a and t-SNARE Stx3 is disrupted upon the loss of Munc18 (gMunc18).

(B) Reciprocally, Stx3 does not pulldown SIp4 in Munc18-KO cells. (C) HS-Munc18_gR interacts with SIp4a and Stx3, while the $\mathrm{d} 232$ mutant abolishes the interaction with $\mathrm{v}$ - and t-SNARE proteins. (A-C) Strep-Tactin was used for coaffinity purification (co-AP). localized preferentially as well at the subapical vesicles and tubules in these FHL5 patient samples (Supplemental Figure 2, D-I), similar to the patterns reported in MVID patients (17). Control samples showed normal marker distribution (Supplemental Figure 2, J-L).

Collectively, mutations in Munc18 in FHL5 patients or loss of Munc18 in vitro resulted in disrupted SNARE-mediated apical fusion of cargo vesicles and, therefore, in a mislocalization of brush-border transporters relevant for physiological enterocyte function (Figure 7). Therefore, we propose that, in addition to the disturbed polarity of patients' enterocytes, e.g., shortening or absence of brush-border microvilli, the disrupted trafficking of brush-border transporters, such as NHE3, might contribute to the enteropathy seen in patients.

\section{Discussion}

FHL5 is a monogenic disorder with hematological and neurological $(11,38)$ symptoms as well as severe watery diarrhea (12). We have focused here on the diarrhea that persists after HSCT (12). This enteropathy bears a wide range of clinical and ultrastructural features of MVID. For a summary of clinical features, mutations, histology, and (immuno)cytochemistry of the FHL5 patients described herein in comparison to MVID, see Supplemental Table 1.

So far, 5 subtypes of FHL have been characterized, each caused by a different dysfunctional gene (2-4, 8-10). However, only disrupted STXBP2 causes MVIDlike symptoms in association with FHL. A potential explanation might be that FHL1-4 genes carry out a biological function that is only required by lymphocytes and leukocytes in order to respond to pathogens. These functions include transport, membrane fusion, and exocytosis of perforin-containing lytic granules.

Yet, the SM protein Munc18 can not only interact with Stx11 (39) (causative for FHL4) and was also shown to interact with Stx3 in polarized epithelial cells (25). SM proteins are required for SNARE-mediated membrane fusion (40). We have previously shown that Munc18 is part of the protein machinery required for fusion of exocytic vesicles with the apical plasma membrane in enterocytes (26).

Analyses of the role of Munc18 in enterocytes allowed us to establish its function within the last steps of apical cargo trafficking toward the brush-border membrane. Munc18 is essential for the fusion of apical cargo vesicles via the v-/t-SNARE-mediated interaction. The Ile232del mutant of Munc18 was shown to disrupt the interaction with Stx11, an interaction relevant for proper leukocyte function (3). Here, we showed that the same mutation also abolished the Munc18-Slp4a/Stx3 interaction, which is pivotal to cargo vesicles fusion with apical plasma membrane. These vesicles are transported by Myo5B associated with small GTPases (e.g., Rab11 and Rab8). The disruption of this pathway reflects the molecular mechanism of MYO5B/STX3 linked MVID (26). Thus, we propose MVIDlinked FHL5 to be based on the same pathophysiological mechanism as MYO5B/STX3 linked MVID.

Ultimately, loss of Munc18 results in a selective cargo trafficking defect. Some apically destined cargo proteins required for enterocyte function, i.e., NHE3 and GLUT5, failed to reach their supposed destination, while the apically destined protein DPPIV was correctly transported to the brush border. The same phenomenon of selectively affected cargo transport/traffic has been observed in certain cases 


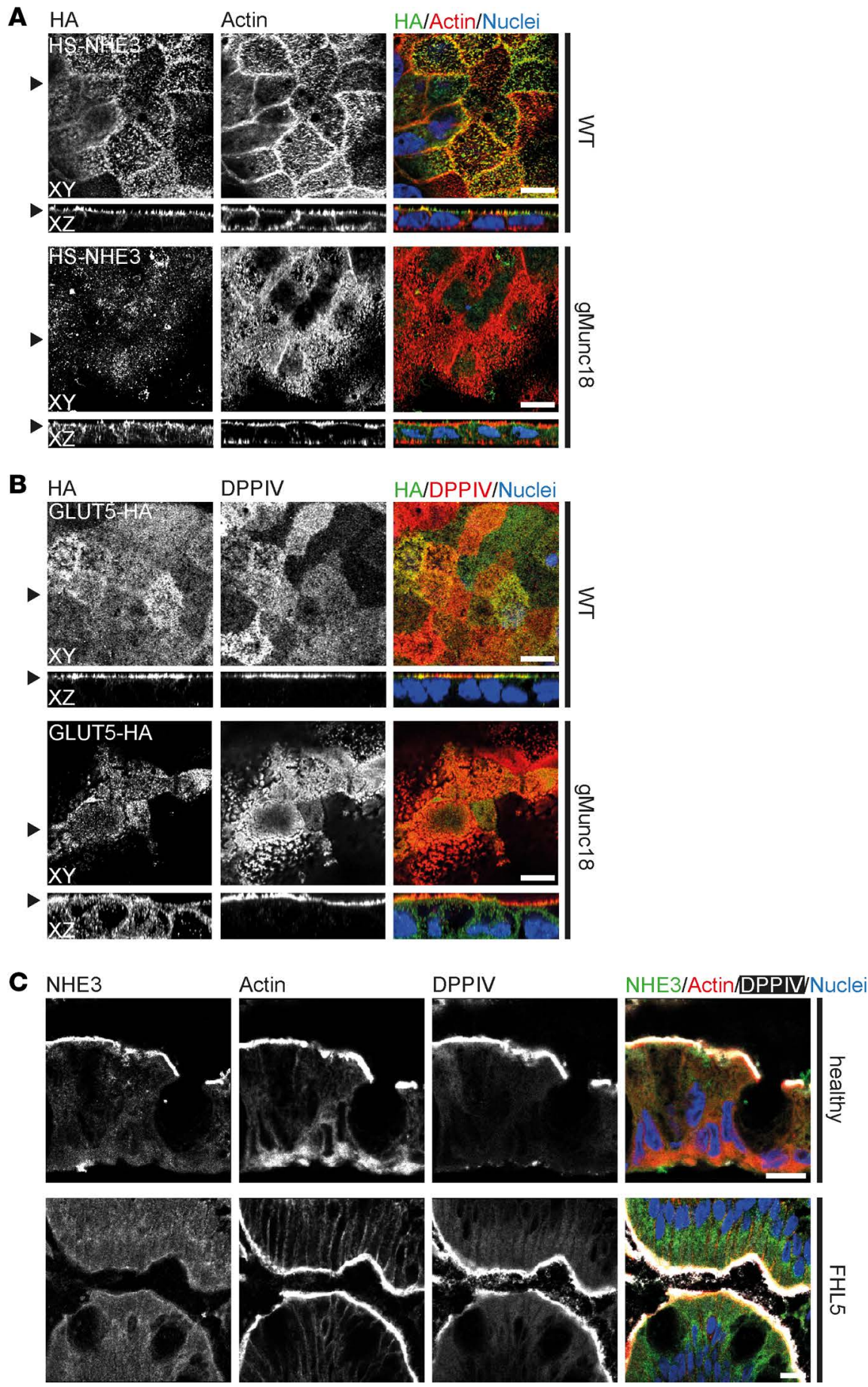

Figure 6. Munc18 is pivotal for selective apical cargo transport of brush-border enzymes. (A) HS-NHE3 is found at the apical brush border in CaCo2 WT cells but redistributes toward the cytoplasm upon Munc18 deletion (gMunc18). (B) Apical GLUT5 mislocalizes in Munc18-KO cells, while DPPIV localizes correctly at the brush border. (C) NHE3 and DPPIV localize to the brush border in healthy duodenal enterocytes. NHE3, but not DPPIV, mislocalizes in FHL5 enterocytes. (A-C) Brush-border microvilli are indicated by actin staining. Scale bar: $10 \mu \mathrm{m}$.

of $M Y O 5 B / S T X 3$-linked MVID (26). This aspect needs further detailed investigation, since other MVID patients showed somehow different localization patterns of apical markers $(41,42)$.

Hematological symptoms of FHL5 can be overcome by HSCT. As every single intestinal crypt comprises its own stem cell niche (43), intestinal symptoms of FHL5, e.g. MVID, can presently not be genetically treated. However, the increased understanding of the pathophysiology of MVID could eventually lead to a novel therapeutic approach. This could be combined with organoid-derived monolayer cultures, as were exemplary generated from the intestinal tissue of patient 3 . This recently introduced technique allows expansion of human intestinal stem cells in vitro and differentiation of these cells into polarized functional intestinal tissue (44). This in vitro tissue could then be further subjected to drug screening, eventually even in combination with genome-editing approaches, in order to identify a potential drug target for the development of therapeutics.

\section{Methods}

Antibodies and reagents. Primary antibodies directed against Actin (WB 1:1,000, MAB1501, Millipore), DPPIV (IF 1:100, HBB3/775/42, DSHB), HA (WB 1:1,000, IF 1:500, MMS-101R, Covance), Munc182 (WB 1:1,000, ab103976, Abcam), NHE3 (IF 1:100, IEM 1:500, HPA036669, Sigma-Aldrich), Rab11a (WB 1:1,000, ab128913, Abcam), Rab11 (IF 1:100, IEM1:30, 71-5300, Life Technologies), Slp4a (WB 1:1,000, HPA001475, Sigma-Aldrich) and Stx3 (WB 1:1,000, IF 1:100, IEM 1:30m, ab133750, Abcam) are commercially available. Secondary horseradish peroxidase-conjugated goat anti-mouse and goat anti-rabbit (1:5,000, Sigma-Aldrich) were used for Western blotting. Actin filaments were labeled with Phalloidin-Alexa Fluor 568 (1:500, Life Technologies). Secondary Alexa Fluor-conjugated (Alexa Fluor 488, 568, and 647) goat antimouse (1:1,000, Life Technologies), goat anti-rabbit (1:1,000, Life Technologies), and Hoechst 3342 (1:10,000, Thermo Scientific) were used for immunofluorescence labeling. For IEM, bound primary antibodies were detected by goat anti-rabbit (Fab' fragment) conjugated to NANOGOLD (2004, Nanoprobes), followed by silver enhancement (2012, HQ-Silver, Nanoprobes). 

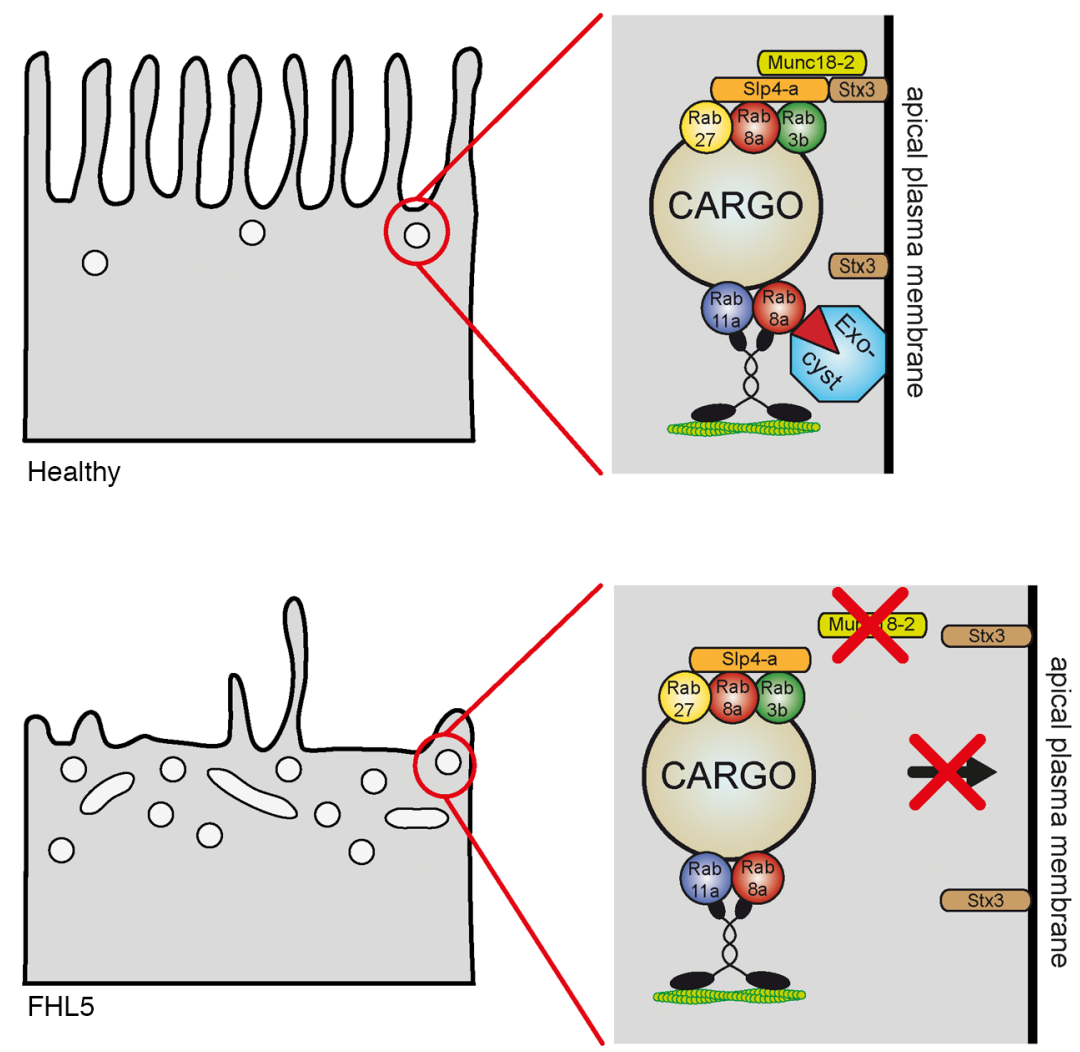

Figure 7. Scheme of the proposed disease mechanism. In healthy enterocytes, cargo vesicles are delivered to the apical plasma membrane and are fused dependently on Munc18 and a previously reported protein machinery (26). When Munc18 is mutated or lost, SNARE-mediated fusion is disturbed and vesicles accumulate subapically.

Genetic analysis. Sanger sequencing of all exons and exon-intron boundaries of the MYO5B gene (encoding myosin $\mathrm{Vb}$ ) was performed for each patient. Results are based on NCBI mRNA reference sequence (NM_001080467; Ensembl ENSG00000167306; Transcript ENST00000285039) (19, 20). Sanger sequencing of all exons and exon-intron boundaries of the STX3 gene (encoding Stx3) was performed for each patient. Results are based on NCBI mRNA reference sequence (NM_004177.4; Ensembl ENSG00000166900; TranscriptENST00000337979) (18).The first nucleotide of the ATG start codon is referred to as nucleotide +1 of the coding sequence.

Whole-exome sequencing was performed with Agilent sureselect xt2 V6 exome enrichment and Illumina HiSeq2500 paired-end sequencing (GATC-Biotech) and a pipeline using Burrows-Wheeler transformation alignment, PICARD PCR duplicates removal and calling of single-nucleotide substitutions and small indels by SAMtools software, and, finally, annotation of variants by use of the SeattleSeqAnnotation server. The annotated variants were filtered against allele frequencies in public databases (Exome aggregation consortium, http://exac.broadinstitute.org/ and the NHLBI Exome Sequencing Project, http:// evs.gs.washington.edu/EVS/) of control exomes and filtered by predicted effects of the variants on their encoded gene products (nonsense, frameshift, missense, etc.) with the help of in silico scores obtained by the PolyPhen2, PhastConservation, and CADD programs.

Whole-exome sequencing data of patient 2 was deposited in a MINSEQE-compliant database (https:// www.ebi.ac.uk/arrayexpress/experiments/E-MTAB-5796).

Genome editing. For CRISPR/Cas9-mediated depletion guide RNA (gRNA), a targeting sequence for Munc18-2 (5'-GAAGACAAGATGCGCATGCT-3') was selected using an online prediction tool (http:// crispr.mit.edu/) (45). gRNAs were cloned into a lentiCRISPRv2 vector via BsmBI restriction enzyme sites. lentiCRISPR v2 was a gift from Feng Zhang (Broad Institute of Massachusetts Institute of Technology [MIT] and Harvard, Cambridge, Massachusetts, USA) (Addgene plasmid 52961) (27). Lentiviral transduction was carried out as described above. Depletion efficiency was verified via Western blotting, and a gRNA-resistant cDNA was used for rescue experiments. 
Plasmids and lentivirus production. Human Munc18-2 was amplified via PCR from cDNA/plasmids, ligated into a pENTR-HA-STREP vector (a gift from Dr. Stephan Geley, [Division of Molecular Pathophysiology, Medical Innsbruck University, Innsbruck, Austria]). The "d232" deletion was introduced via PCR-based mutagenesis. For lentiviral transduction, cDNA was further subcloned via LR clonase into a pCCL-EF1 $\alpha$-BlastiR-DEST lentiviral vector using Gateway Cloning Technology (Invitrogen). EGFP, GLUT5, NHE3, Slp4a, and Stx3 have been described previously (26).

Lentiviral plasmids were cotransfected with Lipofectamine LTX (Invitrogen) together with pVSV-G and psPAX2 in the Hek293LTV producer cell line. Viral supernatant was harvested 48 and 72 hours after transfection and directly used for $\mathrm{CaCo} 2$ cell infection. Six days after infection, cells were selected with 10 $\mu \mathrm{g} / \mathrm{ml}$ Puromycin (Sigma-Aldrich) or $20 \mu \mathrm{g} / \mathrm{ml}$ BlasticidinS (Invitrogen).

Cell culture (cell lines). Hek293LTV (Cell Biolabs), CaCo2 WT (ATCC HTB-37), and KO cells were cultured in Dulbecco's modified Eagle's medium (Sigma-Aldrich) containing high glucose, sodium pyruvate, $100 \mathrm{U} / \mathrm{ml}$ Penicillin (Sigma-Aldrich), $100 \mu \mathrm{g} / \mathrm{ml}$ Streptomycin (Sigma-Aldrich), and 10\% fetal bovine serum (Gibco) in a humidified atmosphere with $5 \% \mathrm{CO}_{2}$ at $37^{\circ} \mathrm{C}$. For experiments requiring fully polarized growth conditions, $\mathrm{CaCo} 2$ cells were seeded on 24-mm Costar Transwell filters (pore size $0.4 \mu \mathrm{m}$, Corning) and cultured for 14-21 days.

Organoid culture. Crypts were isolated from duodenal biopsies, and organoids were generated as described previously (18). The controls were patients suspected of celiac disease with normal pathology. The organoids were maintained in Matrigel (BD Biosciences) with expansion medium as described previously (18), supplemented with $100 \mu \mathrm{g} / \mathrm{ml}$ primocin (InvivoGen) and cultured at $37^{\circ} \mathrm{C}$ and $5 \% \mathrm{CO}_{2}$. Medium was refreshed every 2 or 3 days, and organoids were split every week by mechanical disruption or trypsinization.

To generate a monolayer of polarized enterocytes, organoids were first expanded by regular 3D culture in Matrigel. After 7 days of culture in expansion medium, organoids were harvested and processed into single cells by resuspension and subsequently incubation with $0.25 \%$ trypsin-EDTA (Sigma-Aldrich) for 5-10 minutes. Polyester membrane inserts (6.5-mm Transwell with $0.4-\mu \mathrm{m}$ pore; Corning) were coated for at least 1 hour at room temperature with Matrigel diluted 1:40 in phosphate-buffered salt with calcium chloride and magnesium chloride (Sigma-Aldrich). After removal of Matrigel, $2.5 \times 10^{5}$ to $4.0 \times 10^{5}$ cells were seeded in expansion medium supplemented with Y-27632 (Abcam). Trans-epithelial electrical resistance (TEER) was determined daily by using an epithelial Volt/Ohm meter (World Precision Instruments). After 3 days, the medium was replaced by expansion medium without Y-27632. When cells were confluent and the TEER value reached a plateau phase, usually after another 2 days of culturing, differentiation was induced by replacing the expansion medium with DM (which is expansion medium lacking WNT3A, nicotinamide, and SB202190). When the TEER value reached the next plateau phase, usually after 7 days of culturing in DM, the cells were considered fully differentiated. Fully differentiated organoid-derived monolayers were processed for EM.

Coaffinity purification assays and Western Blotting. For coaffinity purification of HS-tagged proteins, Strep-Tactin purification was carried out as described previously (46). Polarized CaCo2 cells were scraped and lysed in NP40-containing lysis buffer. After separation of nuclei/DNA, lysates were passed by Strep-Tactin resin (IBA Bio TAGnology, 2-1201-010) in columns (Mo Bi Tec Molecular, M1003). Affinity captured proteins were washed and eluted with biotin (46). CaCo2 cells expressing HS-EGFP were used as a control. Western blotting was performed essentially as described before (47). PVDF membranes were incubated with primary antibody at room temperature for 1 hour or overnight at $4^{\circ} \mathrm{C}$. Secondary antibody incubation was carried out for 1 hour at room temperature. Chemiluminescence was exposed on films.

Immunofluorescence microscopy. Immunofluorescence labeling of $\mathrm{CaCo} 2$ cells grown on Transwell filters was carried out essentially as described before (26). 3D cyst cultures were processed as previously described (48) and mounted in Mowiol. Human biopsy material was embedded in Tissue-Tek O.C.T. Compound (Sakura Finetek, VWR) and frozen at $-80^{\circ} \mathrm{C}$ essentially as described previously (31). $10-\mu \mathrm{m}$ cryostat sections were fixed for 20 minutes in $4 \%$ buffered paraformaldehyde solution at room temperature. Samples were analyzed at room temperature with an Axio Imager M1 epifluorescent microscope (Carl Zeiss) equipped with a charge-coupled device camera (SPOT Xplorer; Visitron Systems) and recorded with VisiView 2.0.3 (Visitron Systems). An $\times 10$ air objective lens was used (numerical aperture of 0.3 ) (Carl Zeiss). Single confocal planes or stacks were recorded with a Leica SP5 confocal fluorescence microscope (Leica Microsystems) using a glycerol $\times 63$ lens with a numerical aperture of 1.3 (Leica Microsystems) at room temperature mounted in Mowiol. Recording 
software used was Leica LASAF 2.7.3 (Leica Microsystems). Images were deconvolved with Huygens Professional Deconvolution and AnalysisSoftware (Scientific Volume Imaging), exported using Imaris $3 \mathrm{D}$ rendering (Bitplane AG), and adjusted for brightness, contrast, and pixel size.

Light microscopic PAS cytochemistry. PAS reaction was carried out on paraffin sections or cryostat sections according to standard procedures as previously described (18).

$E M$. Specimen processing was performed essentially as described previously. Briefly, biopsies were chemically fixed with formaldehyde (4\%) and/or glutaraldehyde (2.5\%), optionally followed by osmium tetroxide (0.5\%) (18). CaCo2 cell models were high-pressure frozen and freeze substituted (26). Organoid-derived monolayer cultures were first fixed with formaldehyde, followed by high-pressure freezing and freeze substitution. Semi-thin and ultra-thin resin sections (or cryosections) were analyzed by light microscopy and transmission EM, respectively. PAS-positive material was detected on resin sections according to Thiéry (49) as previously described (26). Immuno-EM for NHE3, Rab11, and Stx3 was performed on thawed cryosections according to Tokuyasu (50) as described previously (17).

Software. If not already specified, we used the following software: GNU Image Manipulation Program, version 2.8.10 (GIMP) open-source software; ImageJ, version 1.49a (NIH), Adobe Photoshop CS6; Adobe Illustrator CS6; and iTEM-analySIS five software (OSIS).

Statistics. Cyst quantification represents the mean of $n \geq 100$ cysts per experiment of at least 3 independent experiments. Error bars represent the SD. Significance was analyzed applying unpaired and 2-tailed Student's $t$ test. $P$ values of less than 0.05 were considered significant.

Study approval. Written informed consent was obtained from the patients' parents, and snap biopsies from small intestinal sites were obtained. Written informed consent for testing of MVID genes or genomewide testing was obtained from the patients' parents following genetic counseling. The study was approved by the respective ethics committees of the universities involved (Medical University Innsbruck, University Medical Centre (UMC) Utrecht, University Medical Center Ulm, Nottingham University Hospitals, Erasmus MC Rotterdam, and Université de Montréal), according to the principles outlined by the Helsinki declaration. Blood samples were obtained for genetic analyses.

\section{Author contributions}

GFV was the leading experimenter and wrote the manuscript. JMVR and IMK contributed organoid cultures. JCE performed experiments. CP, MC, CS, PJ, NP, and ARJ contributed patient materials and genetic analyses. MWH performed transmission EM and contributed to the writing. EC, TM, SM, and LAH designed experiments, supervised the work, and wrote the manuscript.

\section{Acknowledgments}

We thank Angelika Flörl, Karin Gutleben, Caroline Herrmann, Barbara Witting, and Caroline Wiegerinck for technical support and Mariëlle van Gijn for DNA analysis of patient 3. Furthermore, we acknowledge Stephan Geley for providing several plasmids. This work was funded by the Verein zur Förderung der Wissenschaft in Gastroenterologie und Hepatologie Tirol, the Austrian Science Fund (FWF) within the SFB021 special research program and the Molecular Cell Biology and Oncology at the Medical University of Innsbruck PhD program, the Netherlands Organisation for Scientific Research (NWO-ZonMW; VIDI 016.146.353) to SM, and Österreichische Nationalbank Jubiläumsfond grant 16678 to TM.

Address correspondence to: Lukas A. Huber, Division of Cell Biology, Medical University of Innsbruck, Innrain 80-82, 6020 Innsbruck, Austria. Phone: 43.512.9003.70170; Email: lukas.a.huber@i-med.ac.at. Or to: Thomas Müller, Paediatrics I, Medical University of Innsbruck, Anichstrasse 35, 6020 Innsbruck, Austria. Phone: 43.512.504.23501; Email: thomas.mueller@i-med.ac.at. Or to: Sabine Middendorp, UMC Utrecht, Department of Pediatric Gastroenterology, Regenerative Medicine Center Utrecht, Uppsalalaan 8, 3584 CT Utrecht, The Netherlands. Phone: 31.6.18066833; Email: s.middendorp@umcutrecht.nl. Or to: Michael Hess, Division of Histology and Embryology, Medical University of Innsbruck, Müllerstrasse 59, 6020 Innsbruck, Austria. Phone: 43.512.9003.71176; Email: michael.hess@i-med.ac.at.

1. Henter JI, Elinder G, Ost A. Diagnostic guidelines for hemophagocytic lymphohistiocytosis. The FHL Study Group of the Histiocyte Society. Semin Oncol. 1991;18(1):29-33.

2. zur Stadt U, et al. Linkage of familial hemophagocytic lymphohistiocytosis (FHL) type-4 to chromosome 6q24 and identifica- 
tion of mutations in syntaxin 11. Hum Mol Genet. 2005;14(6):827-834.

3. zur Stadt U, et al. Familial hemophagocytic lymphohistiocytosis type 5 (FHL-5) is caused by mutations in Munc18-2 and impaired binding to syntaxin 11. Am J Hum Genet. 2009;85(4):482-492.

4. Côte M, et al. Munc18-2 deficiency causes familial hemophagocytic lymphohistiocytosis type 5 and impairs cytotoxic granule exocytosis in patient NK cells. J Clin Invest. 2009;119(12):3765-3773.

5. Stéphan JL, Donadieu J, Ledeist F, Blanche S, Griscelli C, Fischer A. Treatment of familial hemophagocytic lymphohistiocytosis with antithymocyte globulins, steroids, and cyclosporin A. Blood. 1993;82(8):2319-2323.

6. Mahlaoui N, et al. Immunotherapy of familial hemophagocytic lymphohistiocytosis with antithymocyte globulins: a single-center retrospective report of 38 patients. Pediatrics. 2007;120(3):e622-e628.

7. Machaczka M, Nahi H, Karbach H, Klimkowska M, Hägglund H. Successful treatment of recurrent malignancy-associated hemophagocytic lymphohistiocytosis with a modified HLH-94 immunochemotherapy and allogeneic stem cell transplantation. Med Oncol. 2012;29(2):1231-1236.

8. Ohadi M, et al. Localization of a gene for familial hemophagocytic lymphohistiocytosis at chromosome $9 \mathrm{q} 21.3-22$ by homozygosity mapping. Am J Hum Genet. 1999;64(1):165-171.

9. Stepp SE, et al. Perforin gene defects in familial hemophagocytic lymphohistiocytosis. Science. 1999;286(5446):1957-1959.

10. Feldmann J, et al. Munc13-4 is essential for cytolytic granules fusion and is mutated in a form of familial hemophagocytic lymphohistiocytosis (FHL3). Cell. 2003;115(4):461-473.

11. Pagel J, et al. Distinct mutations in STXBP2 are associated with variable clinical presentations in patients with familial hemophagocytic lymphohistiocytosis type 5 (FHL5). Blood. 2012;119(25):6016-6024.

12. Stepensky P, et al. Persistent defective membrane trafficking in epithelial cells of patients with familial hemophagocytic lymphohistiocytosis type 5 due to STXBP2/MUNC18-2 mutations. Pediatr Blood Cancer. 2013;60(7):1215-1222.

13. Davidson GP, Cutz E, Hamilton JR, Gall DG. Familial enteropathy: a syndrome of protracted diarrhea from birth, failure to thrive, and hypoplastic villus atrophy. Gastroenterology. 1978;75(5):783-790.

14. Cutz E, Rhoads JM, Drumm B, Sherman PM, Durie PR, Forstner GG. Microvillus inclusion disease: an inherited defect of brush-border assembly and differentiation. NEngl J Med. 1989;320(10):646-651.

15. Phillips AD, Schmitz J. Familial microvillous atrophy: a clinicopathological survey of 23 cases. J Pediatr Gastroenterol Nutr. 1992;14(4):380-396.

16. Ruemmele FM, Schmitz J, Goulet O. Microvillous inclusion disease (microvillous atrophy). Orphanet J Rare Dis. $2006 ; 1: 22$.

17. Vogel GF, et al. Abnormal Rab11-Rab8-vesicles cluster in enterocytes of patients with microvillus inclusion disease. Traffic. 2017;18(7):453-464.

18. Wiegerinck CL, et al. Loss of syntaxin 3 causes variant microvillus inclusion disease. Gastroenterology. 2014;147(1):65-68.e10.

19. Müller T, et al. MYO5B mutations cause microvillus inclusion disease and disrupt epithelial cell polarity. Nat Genet. 2008;40(10):1163-1165.

20. Ruemmele FM, et al. Loss-of-function of MYO5B is the main cause of microvillus inclusion disease: 15 novel mutations and a CaCo-2 RNAi cell model. Hum Mutat. 2010;31(5):544-551.

21. van der Velde $\mathrm{KJ}$, et al. An overview and online registry of microvillus inclusion disease patients and their MYO5B mutations. Hum Mutat. 2013;34(12):1597-1605.

22. Bryant DM, Datta A, Rodríguez-Fraticelli AE, Peränen J, Martín-Belmonte F, Mostov KE. A molecular network for de novo generation of the apical surface and lumen. Nat Cell Biol. 2010;12(11):1035-1045.

23. Apodaca G, Gallo LI, Bryant DM. Role of membrane traffic in the generation of epithelial cell asymmetry. Nat Cell Biol. 2012;14(12):1235-1243.

24. Krzewski K, Coligan JE. Human NK cell lytic granules and regulation of their exocytosis. Front Immunol. 2012;3:335.

25. Hackmann Y, et al. Syntaxin binding mechanism and disease-causing mutations in Munc18-2. Proc Natl Acad Sci USA 2013;110(47):E4482-E4491.

26. Vogel GF, Klee KM, Janecke AR, Müller T, Hess MW, Huber LA. Cargo-selective apical exocytosis in epithelial cells is conducted by Myo5B, Slp4a, Vamp7, and syntaxin 3. J Cell Biol. 2015;211(3):587-604.

27. Sanjana NE, Shalem O, Zhang F. Improved vectors and genome-wide libraries for CRISPR screening. Nat Methods. 2014;11(8):783-784

28. Meeths M, et al. Spectrum of clinical presentations in familial hemophagocytic lymphohistiocytosis type 5 patients with mutations in STXBP2. Blood. 2010;116(15):2635-2643.

29. Thoeni CE, et al. Microvillus inclusion disease: loss of Myosin vb disrupts intracellular traffic and cell polarity. Traffic. 2014;15(1):22-42.

30. Knowles BC, et al. Myosin $\mathrm{Vb}$ uncoupling from RAB8A and RAB11A elicits microvillus inclusion disease. J Clin Invest. 2014;124(7):2947-2962.

31. Schneeberger $\mathrm{K}$, et al. An inducible mouse model for microvillus inclusion disease reveals a role for myosin $\mathrm{Vb}$ in apical and basolateral trafficking. Proc Natl Acad Sci USA. 2015;112(40):12408-12413.

32. Iancu TC, Mahajnah M, Manov I, Shaoul R. Microvillous inclusion disease: ultrastructural variability. Ultrastruct Pathol. 2007;31(3):173-188.

33. Fukuda M, Imai A, Nashida T, Shimomura H. Slp4-a/granuphilin-a interacts with syntaxin-2/3 in a Munc18-2-dependent manner. J Biol Chem. 2005;280(47):39175-39184.

34. Schmidt TG, Skerra A. The Strep-tag system for one-step purification and high-affinity detection or capturing of proteins. Nat Protoc. 2007;2(6):1528-1535.

35. Yang J, et al. Rapamycin inhibition of mTOR reduces levels of the $\mathrm{Na}+/ \mathrm{H}+$ exchanger 3 in intestines of mice and humans, leading to diarrhea. Gastroenterology. 2015;149(1):151-162.

36. Yoo BK, Yanda MK, No YR, Yun CC. Human intestinal epithelial cell line SK-CO15 is a new model system to study $\mathrm{Na}(+) / \mathrm{H}(+)$ exchanger 3. Am J Physiol Gastrointest Liver Physiol. 2012;303(2):G180-G188.

37. Uldry M, Thorens B. The SLC2 family of facilitated hexose and polyol transporters. Pflugers Arch. 2004;447(5):480-489.

38. Sandrock K, Nakamura L, Vraetz T, Beutel K, Ehl S, Zieger B. Platelet secretion defect in patients with familial hemophagocyt- 
ic lymphohistiocytosis type 5 (FHL-5). Blood. 2010;116(26):6148-6150.

39. Spessott WA, Sanmillan ML, McCormick ME, Kulkarni VV, Giraudo CG. SM protein Munc18-2 facilitates transition of syntaxin 11-mediated lipid mixing to complete fusion for T-lymphocyte cytotoxicity. Proc Natl Acad Sci U S A 2017;114(11):E2176-E2185.

40. Burkhardt P, Hattendorf DA, Weis WI, Fasshauer D. Munc18a controls SNARE assembly through its interaction with the syntaxin N-peptide. EMBO J. 2008;27(7):923-933.

41. Ameen NA, Salas PJ. Microvillus inclusion disease: a genetic defect affecting apical membrane protein traffic in intestinal epithelium. Traffic. 2000;1(1):76-83.

42. Kravtsov DV, et al. Identification of intestinal ion transport defects in microvillus inclusion disease. Am J Physiol Gastrointest Liver Physiol. 2016;311(1):G142-G155.

43. Barker N. Adult intestinal stem cells: critical drivers of epithelial homeostasis and regeneration. Nat Rev Mol Cell Biol. 2014;15(1):19-33.

44. Sato T, et al. Single Lgr5 stem cells build crypt-villus structures in vitro without a mesenchymal niche. Nature. 2009;459(7244):262-265.

45. Hsu PD, et al. DNA targeting specificity of RNA-guided Cas9 nucleases. Nat Biotechnol. 2013;31(9):827-832.

46. Glatter T, Wepf A, Aebersold R, Gstaiger M. An integrated workflow for charting the human interaction proteome: insights into the PP2A system. Mol Syst Biol. 2009;5:237.

47. Fialka I, Pasquali C, Lottspeich F, Ahorn H, Huber LA. Subcellular fractionation of polarized epithelial cells and identification of organelle-specific proteins by two-dimensional gel electrophoresis. Electrophoresis. 1997;18(14):2582-2590.

48. Jaffe AB, Kaji N, Durgan J, Hall A. Cdc42 controls spindle orientation to position the apical surface during epithelial morphogenesis. J Cell Biol. 2008;183(4):625-633.

49. Thiéry J. Mise en évidence des polysaccharides sur coupes fine en microscopie electronique. J Microscopie. 1967;6: 987-1018

50. Tokuyasu KT. A technique for ultracryotomy of cell suspensions and tissues. J Cell Biol. 1973;57(2):551-565. 